Moroccan J. of Pure and Appl. Anal. (MJPAA)

Volume 8(1), 2022, Pages 22-27

ISSN: Online 2351-8227 - Print 2605-6364

DOI: $10.2478 / \mathrm{mjpaa}-2022-0002$

\title{
Bu's theorem in the positive situation
}

\author{
Amar Bougoutaia $^{1}$ And Amar Belacel ${ }^{2}$
}

AвSTRACT. In this paper, we valorize the relationship between positive $p$-summing operators and positive strongly $q$-summing operators using (Contemp. Math. 328, 145 - 149 (2003)).

Mathematics Subject Classification (2020). 46A20, 47A05, 46B42, 47B10, 47B65.

Key words and phrases. Banach lattice - Positive operators - Positive $p$-summing operators.

\section{Introduction and preliminaries}

Let $E$ be a Banach lattice. We denote by $E^{+}$the positive cone $\{x \in E, x \geq 0\}$. For $x \in E$, let $x^{+}:=\sup \{x, 0\}, x^{-}:=\sup \{-x, 0\}$, be the positive part, the negative part of $x$, respectively. For any $x \in E$, we have $x=x^{+}-x^{-}$and $|x|=x^{+}+x^{-}$.

Let $F$ be a Banach lattice, and let $X$ and $Y$ be Banach spaces. $X^{*}$ denote the topological dual of $X$ and $B_{X}$ denote its closed unit ball.

A linear operator $u: E \longrightarrow F$ is called positive if $u\left(E^{+}\right) \subset F^{+}$.

Let $1 \leq p \leq \infty, p^{*}$ is the conjugate of $p$, i.e., $\frac{1}{p}+\frac{1}{p^{*}}=1$, we denote by $\ell_{p}^{n}(X)$ the space of all sequences $\left(x_{i}\right)_{i=1}^{n}$ in $X$ with the norm

Received January 6, 2021 - Accepted April 16, 2021.

(C) The Author(s) 2021. This article is published with open access by Sidi Mohamed Ben Abdallah University.

${ }^{1}$ Laboratory of Pure and Applied Mathematics (LPAM), University of Laghouat, Laghouat, Algeria.

e-mail: amarbou28@gmail.com (Corresponding Author)

${ }^{2}$ Laboratory of Pure and Applied Mathematics (LPAM), University of Laghouat, Laghouat, Algeria.

e-mail: amarbelacel@yahoo.fr. 


$$
\begin{cases}\left\|\left(x_{i}\right)_{i=1}^{n}\right\|_{p}=\left(\sum_{i=1}^{n}\left\|x_{i}\right\|^{p}\right)^{\frac{1}{p}} ; & 1 \leq p<\infty . \\ \left\|\left(x_{i}\right)_{i=1}^{n}\right\|_{\infty}=\sup _{1 \leq i \leq n}\left\|x_{i}\right\| ; & p=\infty\end{cases}
$$

and by $\ell_{p, w e a k}^{n}(X)$ the space of all sequences $\left(x_{i}\right)_{i=1}^{n}$ in $X$ with the norm

$$
\begin{cases}w_{p}\left(\left(x_{i}\right)_{i=1}^{n}\right)=\left\|\left(x_{i}\right)_{i=1}^{n}\right\|_{\ell_{p, \text { weak }}^{n}(X)}=\sup _{\varphi \in B_{X^{*}}}\left(\sum_{i=1}^{n}\left|\left\langle x_{i}, \varphi\right\rangle\right|^{p}\right)^{\frac{1}{p}} ; & 1 \leq p<\infty . \\ \left\|\left(x_{i}\right)_{i=1}^{n}\right\|_{\ell_{\infty, \text { weak }}^{n}(X)}=\left\|\left(x_{i}\right)_{i=1}^{n}\right\|_{\infty}=\sup _{1 \leq i \leq n}\left\|x_{i}\right\| ; & p=\infty .\end{cases}
$$

Consider the case where $X$ is replaced by a Banach lattice $E$, and define

$$
\ell_{p, \mid \text { weak } \mid}^{n}(E):=\left\{\left(x_{i}\right)_{i=1}^{n}:\left(\left|x_{i}\right|\right)_{i=1}^{n} \in \ell_{p, \text { weak }}^{n}(E)\right\} .
$$

The compact set $B_{E^{*}}^{+}=\left\{\xi \in B_{E^{*}}: \xi \geq 0\right\}=B_{E^{*}} \cap E^{*+}$.

If $x_{1}, \ldots, x_{n} \geq 0$, we have that

$$
\left\|\left(x_{i}\right)_{i=1}^{n}\right\|_{\ell_{p,|w e a k|}^{n}(E)}=\sup _{\xi \in B_{E^{*}}^{+}}\left(\sum_{i=1}^{n}\left\langle x_{i}, \xi\right\rangle^{p}\right)^{\frac{1}{p}}=w_{p}\left(\left(x_{i}\right)_{i=1}^{n}\right) .
$$

Definition 1.1 ([2]). Let $1 \leq p<\infty$. An operator $u: E \rightarrow X$ is said to be positive $p$-summing if there exists a constant $C>0$ such that for all $n \in \mathbb{N}, x_{1}, \ldots, x_{n} \in E$, the inequality

For $p=\infty$

$$
\left\|u\left(x_{i}\right)_{i=1}^{n}\right\|_{p} \leq C\left\|\left(x_{i}\right)_{i=1}^{n}\right\|_{\ell_{p,|w e a k|}^{n}(E)}
$$

$$
\sup _{1 \leq i \leq n}\left\|u\left(x_{i}\right)\right\| \leq C\left\|\left(x_{i}\right)_{i=1}^{n}\right\|_{\ell_{\infty,|w e a k|}^{n}(E)} .
$$

We denote by $\Pi_{p}^{+}(E, X)$, the space of positive $p$-summing operators from $E$ into $X$. $\Pi_{p}^{+}(E, X)$ becomes a Banach space with the norm $\pi_{p}^{+}($.$) , such that \pi_{p}^{+}(u)$ given by the infimum of the constant $C>0$ that verify the inequality (1.1). We have $\Pi_{\infty}^{+}(E, X)=\mathcal{L}(E, X)$.

Definition $1.2([1])$. Let $1 \leq p<\infty$. An operator $u: X \longrightarrow F$ is positive strongly $p$-summing if there exists a constant $C>0$ such that for all $n \in \mathbb{N},\left(x_{i}\right)_{i=1}^{n} \subset X,\left(y_{i}^{*}\right)_{i=1}^{n} \subset F^{*}$, we have

$$
\sum_{i=1}^{n}\left|\left\langle u\left(x_{i}\right), y_{i}^{*}\right\rangle\right| \leq C\left\|\left(x_{i}\right)_{i=1}^{n}\right\|_{p}\left\|\left(y_{i}^{*}\right)_{i=1}^{n}\right\|_{\ell_{p^{*},|w e a k|}^{n}\left(F^{*}\right)} .
$$

The class of all positive strongly $p$-summing operators between $X$ and $F$ is denoted by $\mathcal{D}_{p}^{+}(X, F)$, the infimum of all the constant $C$ in the inequality (1.2) defines a norm $d_{p}^{+}($. on $\mathcal{D}_{p}^{+}(X, F)$.

Formally, the Rademacher functions

$$
r_{n}(t):[0,1] \longrightarrow \mathbb{R}, n \in \mathbb{N}
$$

are defined by setting $r_{n}(t)=\operatorname{sign}\left(\sin 2^{n} \pi t\right)$.

Khinchin's Inequality [6, p 10]. 
For any $0<p<+\infty$, there are positive constants $A_{p}, B_{p}$, such that for any scalars $a_{1}, a_{2}, \ldots, a_{n}$ we have

$$
A_{p} \cdot\left(\sum_{k=1}^{n}\left|a_{k}\right|^{2}\right)^{\frac{1}{2}} \leq\left(\int_{0}^{1}\left|\sum_{k=1}^{n} a_{k} r_{k}(t)\right|^{p} d t\right)^{\frac{1}{p}} \leq B_{p} \cdot\left(\sum_{k=1}^{n}\left|a_{k}\right|^{2}\right)^{\frac{1}{2}} .
$$

Kahane's Inequality [6, p 211].

If $0<p, q<+\infty$, then there is a constant $K_{p, q}>0$ for which

$$
\left(\int_{0}^{1}\left\|\sum_{k=1}^{n} r_{k}(t) x_{k}\right\|^{q} d t\right)^{\frac{1}{q}} \leq K_{p, q}\left(\int_{0}^{1}\left\|\sum_{k=1}^{n} r_{k}(t) x_{k}\right\|^{p} d t\right)^{1 / p} \text {. }
$$

\section{The main result}

In [3], Q. Bu generalized Theorem 4.2.2 of [5] for all $p$ and $q(1<p, q<+\infty)$, i.e. $\Pi_{p}(H, Y) \subset$ $\mathcal{D}_{q}(H, Y)$, where $H$ is a Hilbert space. In this section, we compare the space of positive $p$-summing operators and that of positive strongly $q$-summing operators by generalizing the last result to the positive situation. We prove that:

Theorem 2.1 Let $1<p, q<+\infty$; and let $H$ be a Hilbert space. Then

$$
\Pi_{p}^{+}(H, F) \subset \mathcal{D}_{q}^{+}(H, F) .
$$

Proof. First consider $H=\ell_{2}^{n}(n \in \mathbb{N})$. Let $u \in \Pi_{p}^{+}(H, F)$. By Pietsch's domination theorem [1, Proposition 3.4] there exists a probability measure $\mu$ on the set $B_{\ell_{2}^{n}}^{+}$provided with the weak* topology, and a constant $C>0$, such that for any $x \in \ell_{2}^{n}$

$$
\|u(x)\| \leq \pi_{p}^{+}(u)\left(\int_{B_{\ell_{2}^{n}}^{+}}\left\langle|x|, x^{*}\right\rangle^{p} d \mu\left(x^{*}\right)\right)^{\frac{1}{p}} .
$$

Now for $x_{1}, \ldots, x_{m} \in \ell_{2}^{n}$ and $y_{1}^{*}, \ldots, y_{m}^{*} \in F^{*}$, we have

$$
x_{k}=\sum_{i=1}^{n} x_{k, i} e_{i}, \quad k=1,2, \ldots, m
$$

Then

$$
\begin{aligned}
\sum_{k=1}^{m} \mid & \left\langle u\left(x_{k}\right), y_{k}^{*}\right\rangle\left|=\sum_{k=1}^{m}\right|\left\langle u\left(x_{k}\right), y_{k}^{*+}-y_{k}^{*-}\right\rangle \mid \\
& =\sum_{k=1}^{m}\left|\left\langle\sum_{i=1}^{n} x_{k, i} u\left(e_{i}\right), y_{k}^{*+}-y_{k}^{*-}\right\rangle\right| \\
& \leq \sum_{k=1}^{m}\left(\left|\left\langle\sum_{i=1}^{n} x_{k, i} u\left(e_{i}\right), y_{k}^{*+}\right\rangle\right|+\left|\left\langle\sum_{i=1}^{n} x_{k, i} u\left(e_{i}\right), y_{k}^{*-}\right\rangle\right|\right) \\
& \leq \sum_{k=1}^{m}\left(\sum_{i=1}^{n}\left(x_{k, i}\right)^{2}\right)^{\frac{1}{2}}
\end{aligned}
$$




$$
\times\left[\left(\sum_{i=1}^{n}\left|\left\langle u\left(e_{i}\right), y_{k}^{*+}\right\rangle\right|^{2}\right)^{\frac{1}{2}}+\left(\sum_{i=1}^{n}\left|\left\langle u\left(e_{i}\right), y_{k}^{*-}\right\rangle\right|^{2}\right)^{\frac{1}{2}}\right],
$$

by Khinchin's inequality,

$$
\begin{aligned}
& \leq \sum_{k=1}^{m}\left\|x_{k}\right\| \cdot \frac{1}{A_{q^{*}}}\left(\int_{0}^{1}\left|\sum_{i=1}^{n} r_{i}(t)\left\langle u\left(e_{i}\right), y_{k}^{*+}\right\rangle\right|^{q^{*}} d t\right)^{\frac{1}{q^{*}}} \\
& +\sum_{k=1}^{m}\left\|x_{k}\right\| \frac{1}{A_{q^{*}}^{\prime}}\left(\int_{0}^{1}\left|\sum_{i=1}^{n} r_{i}(t)\left\langle u\left(e_{i}\right), y_{k}^{*-}\right\rangle\right|^{q^{*}} d t\right)^{\frac{1}{q^{*}}} \\
& \leq \frac{1}{A_{q^{*}}}\left(\sum_{k=1}^{m}\left\|x_{k}\right\|^{q}\right)^{\frac{1}{q}} \cdot\left(\sum_{k=1}^{m} \int_{0}^{1}\left|\sum_{i=1}^{n} r_{i}(t)\left\langle u\left(e_{i}\right), y_{k}^{*+}\right\rangle\right|^{q^{*}} d t\right)^{\frac{1}{q^{*}}} \\
& +\frac{1}{A_{q^{*}}^{\prime}}\left(\sum_{k=1}^{m}\left\|x_{k}\right\|^{q}\right)^{\frac{1}{q}} \cdot\left(\sum_{k=1}^{m} \int_{0}^{1}\left|\sum_{i=1}^{n} r_{i}(t)\left\langle u\left(e_{i}\right), y_{k}^{*-}\right\rangle\right|^{q^{*}} d t\right)^{\frac{1}{q^{*}}} \\
& =\left\|\left(x_{k}\right)_{1}^{m}\right\|_{q} \frac{1}{A_{q^{*}}}\left(\int_{0}^{1} \sum_{k=1}^{m}\left|\left\langle\sum_{i=1}^{n} r_{i}(t) u\left(e_{i}\right), y_{k}^{*+}\right\rangle\right|^{q^{*}} d t\right)^{\frac{1}{q^{*}}} \\
& +\left(\sum_{k=1}^{m}\left\|x_{k}\right\|^{q}\right)^{\frac{1}{q}} \frac{1}{A_{q^{*}}^{\prime}}\left(\sum_{k=1}^{m} \int_{0}^{1}\left|\sum_{i=1}^{n} r_{i}(t)\left\langle u\left(e_{i}\right), y_{k}^{*-}\right\rangle\right|^{q^{*}} d t\right)^{\frac{1}{q^{*}}} \\
& =\left(\sum_{k=1}^{m}\left\|x_{k}\right\|^{q}\right)^{\frac{1}{q}} \frac{1}{A_{q^{*}}}\left(\sum_{k=1}^{m} \int_{0}^{1}\left|\sum_{i=1}^{n} r_{i}(t)\left\langle u\left(e_{i}\right), y_{k}^{*+}\right\rangle\right|^{q^{*}} d t\right)^{\frac{1}{q^{*}}} \\
& +\left\|\left(x_{k}\right)_{1}^{m}\right\|_{q} \frac{1}{A_{q^{*}}^{\prime}}\left(\int_{0}^{1} \sum_{k=1}^{m}\left|\left\langle\sum_{i=1}^{n} r_{i}(t) u\left(e_{i}\right), y_{k}^{*-}\right\rangle\right|^{q^{*}} d t\right)^{\frac{1}{q^{*}}} \\
& \leq\left\|\left(x_{k}\right)_{1}^{m}\right\|_{q} \frac{1}{A_{q^{*}}}\left(\int_{0}^{1}\left(\left\|\sum_{i=1}^{n} r_{i}(t) u\left(e_{i}\right)\right\| \cdot\left\|y_{k}^{*+}\right\|_{\ell_{q^{*}, \mid \text { weak } \mid}\left(F^{*}\right)}\right)^{q^{*}} d t\right)^{\frac{1}{q^{*}}} \\
& +\left\|\left(x_{k}\right)_{1}^{m}\right\|_{q} \frac{1}{A_{q^{*}}^{\prime}}\left(\int_{0}^{1}\left(\left\|\sum_{i=1}^{n} r_{i}(t) u\left(e_{i}\right)\right\| \cdot\left\|y_{k}^{*-}\right\|_{\ell_{q^{*}, \mid \text { weak } \mid}\left(F^{*}\right)}\right)^{q^{*}} d t\right)^{\frac{1}{q^{*}}} \\
& =\left\|\left(x_{k}\right)_{1}^{m}\right\|_{q}\left(\int_{0}^{1}\left\|\sum_{i=1}^{n} r_{i}(t) u\left(e_{i}\right)\right\|^{q^{*}} d t\right)^{\frac{1}{q^{*}}} \\
& \times\left(\frac{1}{A_{q^{*}}}\left\|y_{k}^{*+}\right\|_{\ell_{q^{*}, \mid \text { weak } \mid}\left(F^{*}\right)}+\frac{1}{A_{q^{*}}^{\prime}}\left\|y_{k}^{*-}\right\|_{\ell_{q^{*}, \mid \text { weak } \mid}\left(F^{*}\right)}\right) .
\end{aligned}
$$

Consider $\frac{1}{A_{q^{*}}^{\prime \prime}}=\max \left\{\frac{1}{A_{q^{*}}}, \frac{1}{A_{q^{*}}^{\prime}}\right\}$, then 


$$
\sum_{k=1}^{m}\left|\left\langle u\left(x_{k}\right), y_{k}^{*}\right\rangle\right| \leq \frac{2}{A_{q^{*}}^{\prime \prime}}\left\|\left(x_{k}\right)_{1}^{m}\right\|_{q}\left(\int_{0}^{1}\left\|\sum_{i=1}^{n} r_{i}(t) u\left(e_{i}\right)\right\|^{q^{*}} d t\right)^{\frac{1}{q^{*}}}\left\|y_{k}^{*}\right\|_{\ell_{q^{*}, \mid w e a k}\left(F^{*}\right)} .
$$

Now by Kahane's inequality and (2.1), we have

$$
\begin{aligned}
& \left(\int_{0}^{1}\left\|\sum_{i=1}^{n} r_{i}(t) u\left(e_{i}\right)\right\|^{q^{*}} d t\right)^{\frac{1}{q^{*}}} \leq K_{p, q^{*}} \cdot\left(\int_{0}^{1}\left\|\sum_{i=1}^{n} r_{i}(t) u\left(e_{i}\right)\right\|^{p} d t\right)^{\frac{1}{p}} \\
& \leq K_{p, q^{*} \cdot \pi_{p}^{+}}(u) \cdot\left(\int_{0}^{1}\left(\int_{B_{\ell_{2}^{n}}^{+}}\left\langle\left|\sum_{i=1}^{n} r_{i}(t)\left(e_{i}\right)\right|, x^{*}\right\rangle^{p} d \mu\left(x^{*}\right)\right) d t\right)^{\frac{1}{p}} \\
& \leq K_{p, q^{*}} \cdot \pi_{p}^{+}(u) \cdot\left(\int_{0}^{1}\left(\int_{B_{\ell_{2}^{n}}^{+}}\left\langle\sum_{i=1}^{n}\left|r_{i}(t)\left(e_{i}\right)\right|, x^{*}\right\rangle^{p} d \mu\left(x^{*}\right)\right) d t\right)^{\frac{1}{p}} \\
& \leq K_{p, q^{*} \cdot \pi_{p}^{+}}(u) \cdot\left(\int_{B_{\ell_{2}^{n}}^{+}}\left(\int_{0}^{1}\left\langle\sum_{i=1}^{n}\left|r_{i}(t)\left(e_{i}\right)\right|, x^{*}\right\rangle^{p} d t\right) d \mu\left(x^{*}\right)\right)^{\frac{1}{p}},
\end{aligned}
$$

by Khinchin's inequality

$$
\begin{aligned}
& \left(\int_{0}^{1}\left\|\sum_{i=1}^{n} r_{i}(t) u\left(e_{i}\right)\right\|^{q^{*}} d t\right)^{\frac{1}{q^{*}}} \\
& \leq K_{p, q^{*}} \cdot \pi_{p}^{+}(u) \cdot\left(\int_{B_{\ell_{2}^{n}}^{+}}\left(B_{p}\left(\sum_{i=1}^{n}\left\langle e_{i}, x^{*}\right\rangle^{2}\right)^{\frac{1}{2}}\right) d \mu\left(x^{*}\right)\right)^{\frac{1}{p}} \\
& \leq K_{p, q^{*}} \cdot \pi_{p}^{+}(u) \cdot B_{p}\left(\int_{B_{\ell_{2}^{n}}^{+}}\left\|x^{*}\right\|^{p} d \mu\left(x^{*}\right)\right)^{\frac{1}{p}} \text {, }
\end{aligned}
$$

then

$$
\left(\int_{0}^{1}\left\|\sum_{i=1}^{n} r_{i}(t) u\left(e_{i}\right)\right\|^{q^{*}} d t\right)^{\frac{1}{q^{*}}} \leq K_{p, q^{*}} \pi_{p}^{+}(u) B_{p} .
$$

Combining (2.2) and (2.3)

$$
\sum_{k=1}^{m}\left|\left\langle u\left(x_{k}\right), y_{k}^{*}\right\rangle\right| \leq \frac{2}{A_{q^{*}}^{\prime \prime}} K_{p, q^{*}} \cdot \pi_{p}^{+}(u) \cdot B_{p}\left\|\left(x_{k}\right)_{1}^{m}\right\|_{q}\left\|y_{k}^{*}\right\|_{\ell_{q^{*},|w e a k|}\left(F^{*}\right)} .
$$

So by [1, Definition 4.1], we obtain $u \in \mathcal{D}_{q}^{+}\left(\ell_{2}^{n}, F\right)$ with

$$
d_{q}^{+}(u) \leq \frac{2}{A_{q^{*}}^{\prime \prime}} K_{p, q^{*}} \cdot B_{p} \cdot \pi_{p}^{+}(u) .
$$

Now, consider a general Hilbert space $H$. Let $x_{1}, \ldots, x_{m} \in H$ and $y_{1}^{*}, \ldots, y_{m}^{*} \in F^{*}$. Then there is an $n \in \mathbb{N}$ such that span $\left\{x_{k}\right\}_{k=1}^{m}$ is isometrically isomorphic to $\ell_{2}^{n}$. Let $P$ be the orthogonal projection from $H$ onto span $\left\{x_{k}\right\}_{k=1}^{m}$. 
Then by (2.4)

$$
\begin{aligned}
\sum_{k=1}^{m}\left|\left\langle u\left(x_{k}\right), y_{k}^{*}\right\rangle\right| & =\sum_{k=1}^{m}\left|\left\langle u\left(P\left(x_{k}\right)\right), y_{k}^{*}\right\rangle\right| \\
& \leq \frac{2}{A_{q^{*}}^{\prime \prime}} K_{p, q^{*} \cdot \pi_{p}^{+}}(u) \cdot B_{p}\left\|P\left(x_{k}\right)_{1}^{m}\right\|\left\|_{q}\right\| y_{k}^{*} \|_{\ell_{q^{*}, \mid \text { weak } \mid}\left(F^{*}\right)} . \\
& =\frac{2}{A_{q^{*}}^{\prime \prime}} K_{p, q^{*}} \cdot \pi_{p}^{+}(u) \cdot B_{p}\left\|\left(x_{k}\right)_{1}^{m}\right\|\left\|_{q}\right\| y_{k}^{*} \|_{\ell_{q^{*}, \mid \text { weak } \mid}\left(F^{*}\right)} .
\end{aligned}
$$

This shows us that $u \in \mathcal{D}_{q}^{+}(H, F)$ with

$$
d_{q}^{+}(u) \leq \frac{2}{A_{q^{*}}^{\prime \prime}} K_{p, q^{*}} \cdot B_{p} \cdot \pi_{p}^{+}(u) \text {. }
$$

\section{Acknowledgments}

The authors are grateful to an anonymous referee for many important suggestions and guidelines to make this paper more coherent. The authors acknowledge with thanks the support of the General Direction of Scientific Research and Technological Development (DGRSDT), Algeria.

\section{References}

[1] Achour, D., Belacel, A., Domination and factorization theorems for positive strongly $p$-summing operators. Positivity. 18(2014), $785-804$.

[2] Blasco, O., A class of operators from a Banach lattice into a Banach space. Collect. Math, 37(1)(1986), 13 - 22.

[3] $\mathrm{Bu}, \mathrm{Q}$., Some mapping properties of $p$-summing operators with Hilbertian domain. Contemp. Math. $328(2003), 145-149$.

[4] Bu, Q., Buskes, G., The Radon-Nikodym property for tensor products of Banach lattices. Positivity. 10(2)(2006), $365-390$.

[5] Cohen, J.S., Absolutely p-summing, p-nuclear operators and their conjugates. Math. Ann. 201(1973), 177 - 200.

[6] Diestel, J., Jarchow, H., Tonge, A., Absolutely Summing Operators. Cambridge University Press, Cambridge. (1995). 